\title{
Author Correction: Efficacy and safety assessment of a TRAF6-targeted nanoimmunotherapy in atherosclerotic mice and non-human primates
}

Marnix Lameijer, Tina Binderup, Mandy M. T. van Leent, Max L. Senders, Francois Fay, Joost Malkus, Brenda L. Sanchez-Gaytan, Abraham J. P. Teunissen, Nicolas Karakatsanis (D, Philip Robson, Xianxiao Zhou, Yuxiang Ye, Gregory Wojtkiewicz (D), Jun Tang (D), Tom T. P. Seijkens, Jeffrey Kroon (D), Erik S. G. Stroes, Andreas Kjaer (iD, Jordi Ochando, Thomas Reiner (D), Carlos Pérez-Medina, Claudia Calcagno, Edward A. Fisher, Bin Zhang, Ryan E. Temel, Filip K. Swirski, Matthias Nahrendorf (D, Zahi A. Fayad, Esther Lutgens, Willem J. M. Mulder and Raphaël Duivenvoorden

Correction to: Nature Biomedical Engineering https://doi.org/10.1038/s41551-018-0221-2, published online 16 April 2018.

In the version of this Article originally published, the surname of the author Edward A. Fisher was spelt incorrectly as 'Fischer'. This has now been corrected. 